Ricans, diluting the overall rates of disordor within their particular class/minority combination.

Evidenco from the authors' own research is still exploratory and ambiguous; the conceptual refinement of their approach nonetheless warrants careful consideration by anybody concerned with these problems.

\section{Peter Kelvin}

\section{RADIATION PROTECTION}

\section{Basic Radiation Protection}

Principles and Organization. By C. W. Easloy. Pp. 132. (Gordon and Breach: New York and London, October 1969.) $125 s ; \$ 15$.

IT is very difficult to decide what basis was used for the selection of the material for this little book. In his preface, the author states that the book eontains the information which has been useful to him and that he felt the desire to pass it on. The publishers obviously thought very highly of this collection of notes and happily charge accordingly.

The anthor covors the field in ten chapters and there are thirt pages of appendices. The equipment described is usually commercially available in the United States. The style of writing is certainly not verbose and the layout of the chapters is systematic. The brevity in the technical descriptions is good, but any background information suffers from it. Appendix A contains about thirty entries of two to three lines each. One finds definitions, for instance, for the atorn, dosimetry, electron, molecule, roentyen, rern, but not for the rad, which I would have thought was most important. Appendix B contains sixteen full-page facsimile reproductions of forms of the type used in many laboratories handling radioactive material and usually designed by the laboratories accord. ing to their own needs. The literature of this large ficld is corered by five sources of information; the most recent one is a textbook published in 1960. It is surprising that the publishers believe they can find a market among scientists for this expensive little book and that they believe readers of Nature might be at all interested.

\section{EBERT}

\section{CHOQUET'S THEORY}

\section{Lectures on Analysis}

By Gustave Choquet. Edited by J. Marsden, T. Lance and S. Gelbart. Vol. I: Integration and Topological Vector Spaces. Pp. xix +382 . Vol. 2: Representation Theory. Pp. xix +336 . Vol. 3: Infinite Dimensional Measures and Problem Solutions. Pp. xix +34.1. (W. A. Benjamin: New York and Arnsterdam, 1969.) Cloth $\$ 12.50$ each; paper $\$ 4.95$ each.

This book is an edited account of a graduate course of lectures delivered at Princeton by Professor Choquet in the fall term of 1967. It is a notable addition to the large number of books on functional analysis, chiefly because it provides the most complete account to date of what is justly called Choquet representation theory.

The first half of the course was an introduction to general topology, integration and topological vector spaces. The reader is supposed to know the rudiments of topology and conventional (set-function) measure theory: what is needed here is summarized without proof. The course proper opens with some lectures on selected topics from general topology: nots, filters, uniform spaces, embedding and metrization, and Baire spaces.

The treatment of Baire spaces is original in its use of the notion of a winning tactic from the theory of games. Two players, $\alpha, \beta$, play a certain game with the open sets and points of a topological space. The latter is called $\alpha$-favourable if $\alpha$ has a winning tactic: this is sufficient for the space to be a Baire space. Besides being elegant, the notion of an $\alpha$-favourable space is more pliable than that of a Baire space. For instance, it survives the formation of topological products, which is more than can be said (it is an open question) of the property of being a metric Baire space. 'The use of Baire spaces in analysis is illustrated by a wide variety of examples.

There is a substantial introduction to Bourbaki integration theory in later lectures. This opens with an account of Choquet's theory of capacities, which, with the Reisz ropresentation theorem, serves inter alia to link conventional measure theory and the Bourbaki theory.

There follows an introduction to topological vector spaces, with some emphasis on convexity and partially ordered vector spaces.

Having thus introduced the reader to many of the basic tools and concepts of functional analysis, the author has also set the stage for Choquet representation theory, which occupies most of the second half of this work.

According to the Krein-Milman theorem, each noncmpty compact convex set $K$ in a Hausdorff locally convex space is the closed convex hull of its extreme points. Every point of $K$ then is a limit of convex combinations of extreme points. Can the limit always be realized by an integration? This is the question that Choquet theory, in its original form, answered affirmatively: every point of $K$ is the barycentre of a probability measure that is carried, in a certain sense, by the extrome points of $h$. Early versions of this theorem appeared in 1956, and the basic theory was perfected (by the joint efforts of a number of mathematicians) by the early 1960s. Since then it has been much developed, and this account shows us the state of the subject in 1967.

The theory is applied here to prove Bochner's theorem, Bernstein's theorem on completely monotone functions, and the ergodic decomposition of invariant measures. Other applications to potential theory and operator theory are sketched (much work has been done in these two ficlds, and in simplex theory, since these lectures were given).

The work concludes with a treatment of measures in infinite-dimensional vector spaces which includes a proof, by use of Choquet theory, of the Umemura-Choquet theorem that every rotation-invariant affine measure in an infinite-dimensional pre-Hilbert space is an average of Wiener measures. There is an appendix on potential theory.

The book omits certain proofs that were omitted by the lecturer, but in such cases references to standard works are usually given. There is a large collection of problems, many of them solved in an appendix. There are a good many minor misprints. All foreign words are printed withont diacritical marks. D. A. EDWards

\section{CLASSIC ASTRONOMY}

\section{Celestial Mechanics}

Vol. 5. By the Marquis de la Place. In French. Pp. ix +508 . (Chelsea: Bronx, New York, 1969. First. published 1825.) \$17.50.

THE reprint of Bowditch's English translation of Laplaco's Mécanique Céleste has now been completed by a reprint of the fifth volume of Laplace's great work, which Bowditch did not manage to translate. In this volume, which was published many years later than the main body of his work, Laplace collected various supploments to the matters treated in the previous volumes. For us, however, the chief interest of the fifth volume lies in the historical sections, in which Laplace gives, for each topic, a critical account of the work preceding his own. Taken together, these sections present an outline of the whole progress of theoretical astronomy, distinguished by exquisite precision and unequalled mastery in bringing; 